\title{
Clinical study on angiotensin II vaccination-the first big step
}

\author{
Masaki Mogi ${ }^{1}$
}

Received: 21 September 2021 / Accepted: 1 October 2021 / Published online: 27 October 2021

(c) The Japanese Society of Hypertension 2021

Hypertension is a major cause of premature death worldwide. The number of individuals with hypertension is estimated to be up to 1.28 billion, and two-thirds of them live in low- and middle-income countries (LMICs) [1]. Moreover, the number of individuals with hypertension is increasing in LMICs mainly due to an increase in hypertension risk factors such as changes in diet and salt intake. However, the management of hypertension in LMICs is not satisfactory because of low hypertension awareness, a lack of adequate access to essential medications and nonadherence to antihypertensive medication (Fig. 1) [2].

Vaccines are relatively easy to deliver and provide lifelong protection in most cases. Thus, vaccination to prevent hypertension may help people with lower cardiovascular risk living in LMICs. The present study by Nalagami et al. was a phase I/IIa study of the angiotensin II vaccine AGMG0201 in patients with mild-to-moderate essential hypertension [3]. AGMG0201 vaccination was well tolerated at both low and high doses. A measurable antibody titer was detected in most individuals, but the antibody titer varied among individuals. No severe adverse effects were observed in the vaccination groups, especially in the highdose group. The efficacy of this vaccine for blood pressure was not investigated in this study. Next, advanced clinical studies to investigate the efficacy of this vaccine are expected.
Fig. 1 Potential of vaccination against hypertension

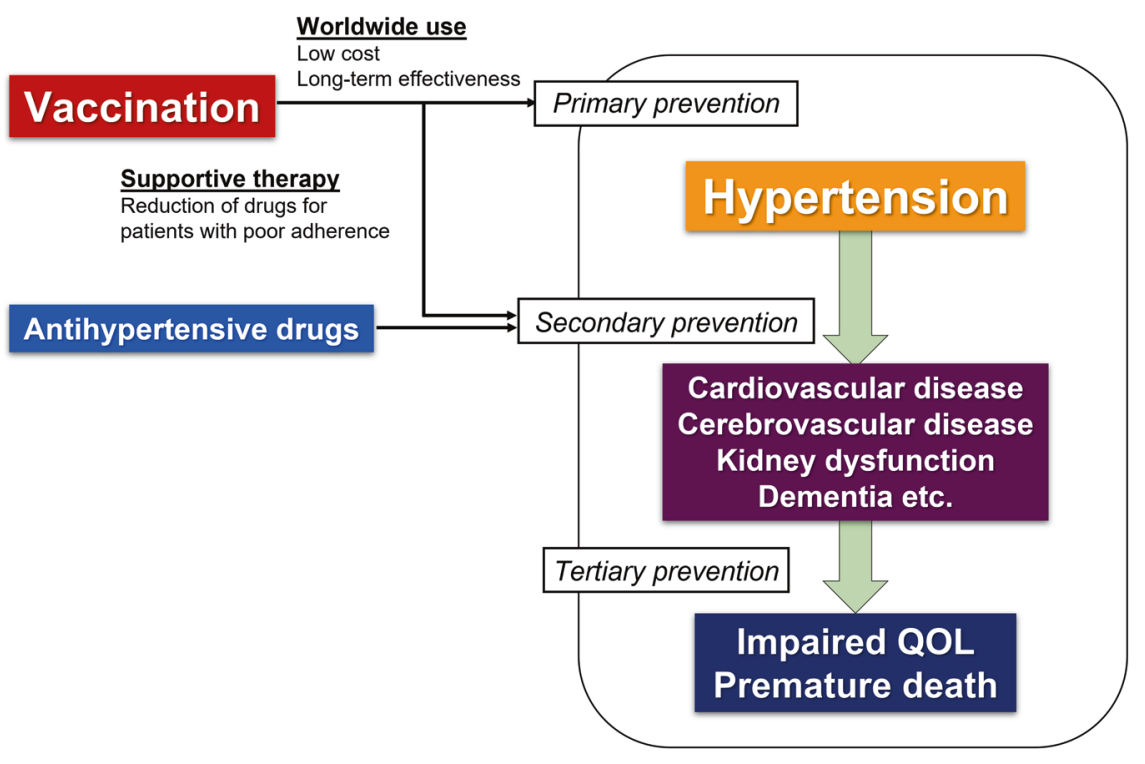

Masaki Mogi

mmogi@m.ehime-u.ac.jp

1 Department of Pharmacology, Ehime University, Graduate School of Medicine, Shitsukawa, Tohon, Ehime 791-0295, Japan 
Vaccines against renin-angiotensin system (RAS) components such as renin, angiotensin I, angiotensin II, and angiotensin II type 1 receptor are well summarized by Garay-Gutiérrez et al. [4]. Nakagami et al. have also clearly and briefly summarized potential therapeutic vaccines for hypertension in the last 20 years in Hypertension Research [5]. In this review, Nakagami et al. show a schematic presentation of the therapeutic vaccine mechanism against selfantigen and introduce a presently ongoing trial of this DNA vaccine. Nakagami et al. also suggest that therapeutic vaccination for lifestyle-related diseases such as hypertension, diabetes, or dyslipidemia is expected to be a new alternative to daily medication as a maintenance tool over a long period.

One of the most well-known angiotensin II vaccines is AngQb-Cyt006, which was investigated in a phase IIa trial in 72 patients with mild-to-moderate hypertension [6]. Those in the high-dose group showed a reduction in blood pressure, especially in the early morning. However, phase III studies of AngQb-Cyt006 were not performed because of its lower antihypertensive effects compared to existing RAS inhibitors [7]. The present study by Nakagami et al. is the first step in the application of this AGMG0201 vaccine. There may be many challenges to overcome before its use in clinical settings. For example, in the present study, some individuals had a high titer of angiotensin II antibody, even in the lowdose group. An investigation of why these participants acquired a high antibody titer may help optimize the efficacy of the vaccine. The efficacy of this angiotensin
II vaccine for blood pressure reduction is expected to be investigated next.

\section{Compliance with ethical standards}

Conflict of interest The author declares no competing interests.

Publisher's note Springer Nature remains neutral with regard to jurisdictional claims in published maps and institutional affiliations.

\section{References}

1. Fact sheets, Hypertension. 2021. https://www.who.int/news-room/ fact-sheets/detail/hypertension.

2. Abegaz TM, Shehab A, Gebreyohannes EA, Bhagavathula AS, Elnour AA. Nonadherence to antihypertensive drugs: a systematic review and meta-analysis. Medicine. 2017;96:e5641.

3. Nakagami H, Ishihama T, Daikyoji Y, Sasakura C, Yamada E, Morishita R. Brief report on a phase I/IIa study to assess the safety, tolerability, and immune response of AGMG0201 in patients with essential hypertension. Hypertens Res. 2021. https://doi.org/10. 1038/s41440-021-00755-6.

4. Garay-Gutierrez NF, Hernandez-Fuentes CP, Garcia-Rivas G, Lavandero S, Guerrero-Beltran CE. Vaccines against components of the renin-angiotensin system. Heart Fail Rev. 2021;26:711-26.

5. Nakagami H, Hayashi H, Shimamura M, Rakugi H, Morishita R. Therapeutic vaccine for chronic diseases after the COVID-19 Era. Hypertens Res. 2021;44:1047-53.

6. Tissot AC, Maurer P, Nussberger J, Sabat R, Pfister T, Ignatenko S, et al. Effect of immunization against angiotensin II with CYT006AngQb on ambulatory blood pressure: a double-blind, randomized, placebo-controlled phase IIa study. Lancet. 2008;371:821-7.

7. Brown MJ. Success and failure of vaccines against reninangiotensin system components. Nat Rev Cardiol. 2009;6:639-47. 